\title{
Manejo multidisciplinario con terapia de electroestimulación en un paciente con síndrome de Goldenhar, trastorno de la deglución y falla para crecer
}

\author{
Emilio Zenteno-Salazar ${ }^{1}$, Claudia Sifuentes-Vela ${ }^{1,2 *}$, Silvina Contreras-Capetillo ${ }^{3}$, Marisol López-Cabrera ${ }^{4}$ \\ Juan C. Núñez-Enríquez ${ }^{5}$ \\ ${ }^{1}$ Escuela de Ciencias de la Salud, Universidad Marista de Mérida, Mérida, Yucatán; ${ }^{2}$ Gastroenterología y Nutrición Pediátrica, Hospitales Star \\ Médica, Mérida, Yucatán; ${ }^{3}$ Laboratorio de Genética y Salud Reproductiva, Centro de Investigaciones Regionales Dr. Hideyo Noguchi, Universidad \\ Autónoma de Yucatán, Mérida, Yucatán; ${ }^{4}$ Foniatría y Trastornos de Deglución, Voz y Habla, Clínica de Mérida, Mérida, Yucatán; ${ }^{5}$ Unidad de \\ Investigación Médica en Epidemiología Clínica, Unidad Médica de Alta Especialidad Hospital de Pediatría Dr. Silvestre Frenk Freund, Centro \\ Médico Nacional Siglo XXI, Instituto Mexicano del Seguro Social, Ciudad de México. México
}

\begin{abstract}
Resumen
Introducción: El síndrome de Goldenhar es un trastorno heterogéneo, esporádico en su mayoría o por patrón de herencia autosómico dominante o recesivo, de la morfogénesis craneofacial asociada al primero y segundo arcos faríngeos, y forma parte del espectro oculoauriculovertebral. La incidencia es de 1 por cada 3500-45,000 recién nacidos vivos, con una razón de sexo masculino/femenino de 3:2. Caso clínico: Se presenta el caso de un recién nacido con fenotipo de síndrome oculoauriculovertebral. Se abordó con radiografía de tórax, ecografía abdominal y tamizaje metabólico y auditivo, que reportaron hemivértebra torácica, fusión costal, quiste renal e hipoacusia bilateral profunda, respectivamente. Fue alimentado con lactancia mixta desde el nacimiento, sin lograr una succión adecuada y con pérdida de peso. A los 3 meses de edad recibió terapia de rehabilitación oral con electroestimulación en conjunto de 10 sesiones con $10 \mathrm{~mA}$ de intensidad, al igual que a los 23, 24, 25, 27, 30 y 32 meses de edad. A los 4 meses, espesamiento de fórmula con cereal; a los 7 meses, sonda de gastrostomía; a los 20 meses, cirugía de paladar y macrostomía. Mostró mejoría en intensidad de babeo en las primeras 10 sesiones y mejoría en la deglución a las 30 sesiones. A los 3 años de edad consume el 100\% de los alimentos por vía oral. Conclusiones: Con la escasa evidencia científica que este caso aislado aporta, el tratamiento con la terapia de rehabilitación en conjunto con la terapia convencional y la corrección anatómica dio resultados positivos para el trastorno de la deglución.
\end{abstract}

Palabras clave: Síndrome de Goldenhar. Falla para crecer. Espectro oculoauriculovertebral. Electroestimulación. Disfagia.

\section{Multidisciplinary management with neuromuscular electrical stimulation therapy in a Goldenhar syndrome patient who presented swallowing disorder and failure to thrive}

\section{Abstract}

Background: Goldenhar syndrome is a heterogeneous disorder, mostly sporadic or due to a dominant autosomal or recessive pattern of inheritance, that exhibits craniofacial morphogenesis associated with the first and second pharyngeal arches

Correspondencia:

${ }^{\star}$ Claudia Sifuentes-Vela

E-mail: clausifuentes@ hotmail.com
Fecha de recepción: 17-07-2020

Fecha de aceptación: 31-08-2020

DOI: 10.24875/BMHIM.20000222
Disponible en internet: 23-07-2021

Bol Med Hosp Infant Mex. 2021;78(4):362-369

www.bmhim.com

1665-1146/@ 2020 Hospital Infantil de México Federico Gómez. Publicado por Permanyer. Este es un artículo open access bajo la licencia CC BY-NC-ND (http://creativecommons.org/licenses/by-nc-nd/4.0/). 
and is part of the oculoauriculovertebral spectrum. Its incidence is of 1 in 3,500-45,000 live newborns, with a male to female ratio of 3:2. Case report: We describe the case of a male newborn with oculoauriculovertebral syndrome phenotype. It was approached with chest $X$-ray, abdominal ultrasound, metabolic and hearing screening, which reported thoracic hemivertebra, costal fusion, renal cyst, and profound bilateral hypoacusis, respectively. Although the newborn was fed with mixed lactation from birth, adequate suction and with weight loss were not achieved. At 3 months of age, as well as at 23, 24, 25, 27,30 and 32 months of age, the infant received oral rehabilitation therapy with electrostimulation in a set of 10 sessions with $10 \mathrm{~mA}$ intensity. At 4 months, thickening of formula with cereal; at 7 months, gastrostomy tube; at 20 months, palate surgery and macrostomy. Improvement in drooling intensity was observed during the first 10 sessions, and improvement in swallowing after 30 sessions. At 3 years of age, the patient consumes $100 \%$ of food orally. Conclusions: According to limited scientific evidence that this isolated case provides, rehabilitation therapy together with conventional therapy coupled with anatomical correction gave positive results for swallowing disorder.

Keywords: Goldenhar syndrome. Failure to thrive. Oculoauriculovertebral spectrum. Electric stimulation. Dysphagia.

\section{Introducción}

El espectro oculoauriculovertebral, sugerido por Gorlin en 1963, o síndrome de Goldenhar, es un trastorno heterogéneo de la morfogénesis craneofacial asociada al primero y segundo arcos faríngeos cuya etiología y patogénesis aún no están bien documentadas ${ }^{1}$. En la mayoría de las familias solo se encuentra un paciente afectado, por lo que se consideran como casos esporádicos. Sin embargo, se reportan patrones de herencia autosómica dominante y recesiva de acuerdo con el espectro de manifestaciones clíni$\operatorname{cas}^{1,2}$. La incidencia del síndrome de Goldenhar es de 1 por cada 3500-5600 hasta 45,000 recién nacidos vivos, y es más frecuente en el sexo masculino (razón 3:2) $)^{1,3}$.

Entre las malformaciones reportadas del espectro oculoauriculovertebral se encuentran aquellas de las estructuras provenientes del primero y segundo arcos faríngeos, con el siguiente fenotipo: quistes dermoides epibulbares, dacriocistitis, apéndices y fístulas preauriculares, paladar hendido e hipoplasia malar, mandibular y maxilar. Adicionalmente puede haber anormalidades vertebrales, cardiacas, renales, gastrointestinales y del sistema nervioso central (Tabla 1) ${ }^{1-3}$; dentro de este último se reportan anormalidades en los nervios craneales VIII, VII, VI y V, aunque el más afectado es el V con hipoplasia o aplasia ${ }^{3,4}$.

Como consecuencia de las estructuras y de los sistemas afectados, suele presentarse una restricción del crecimiento prenatal y posnatal, así como retraso del neurodesarrollo en las áreas del lenguaje, la audición y la deglución.

El objetivo del presente reporte fue comunicar los resultados del manejo multidisciplinario en un paciente con síndrome de Goldenhar que presentó disfagia orofaríngea y falla para crecer.

\section{Caso clínico}

Se aborda el caso de un recién nacido de sexo masculino con anormalidades craneofaciales, hijo de padres mexicanos, no consanguíneos y sanos. En la evaluación prenatal se documentó polihidramnios, microrretrognatia y apéndices preauriculares bilaterales por ecografía a las 29 semanas de gestación (Fig. 1). Se obtuvo producto vivo por cesárea a las 38 semanas de gestación, con Apgar 8/9, peso de $3040 \mathrm{~g}$ (puntuación Z: 0), longitud de $50 \mathrm{~cm}$ (puntuación Z: 0) y perímetro cefálico de $37 \mathrm{~cm}$ (puntuación Z: +2) (Fig. 2). Cabeza con fontanela anterior normal, asimetría facial, fisuras palpebrales amplias con quiste dermoide epibulbar en el ojo derecho, macrostomía con hendiduras faciales bilaterales, paladar hendido, hipoplasia mandibular, microtia derecha y apéndices preauriculares bilaterales. Cuello sin alteraciones y tórax simétrico con pezones invertidos. La sospecha diagnóstica inicial fue espectro oculoauriculovertebral. Se realizó ultrasonido transfontanelar, sin alteraciones; radiografía de columna, con hemivértebra torácica y fusión de las costillas 5 y 6 del lado derecho; ecocardiograma, sin alteraciones; ultrasonido abdominal, con quiste renal simple derecho; tamizaje neonatal ampliado, sin alteraciones; y tamiz auditivo, con hipoacusia neurosensorial bilateral profunda.

Desde el nacimiento fue alimentado con lactancia mixta, sin lograr una succión adecuada, presentando atragantamiento intermitente y poca ganancia ponderal. A partir del primer mes de edad se comenzó a notar una disminución en la velocidad de crecimiento (Fig. 2). A los 3 meses de edad fue valorado por gastroenterología y nutrición pediátrica con el objetivo de evaluar las estrategias terapéuticas para la alteración de la mecánica de la deglución. La primera intervención fue la instrucción a los padres sobre técnicas de 
Tabla 1. Prevalencia de fenotipos del espectro oculoauriculovertebral ${ }^{1,2}$

\begin{tabular}{|c|c|c|}
\hline Localización & Malformación & Porcentaje \\
\hline \multirow[t]{6}{*}{ Craneofacial } & Microsomía hemifacial & $83^{*}$ \\
\hline & Microcefalia & 8 \\
\hline & Paladar hendido & $20-16^{*}$ \\
\hline & Macrostomía & $15-37^{*}$ \\
\hline & Parálisis del nervio craneal VII & $10-45^{*}$ \\
\hline & Malformaciones odontológicas & * \\
\hline \multirow[t]{5}{*}{ Oído } & Microtia/anotia & $78-90^{*}$ \\
\hline & Apéndices preauriculares & $50-64^{*}$ \\
\hline & Orificio preauricular & $6-8$ \\
\hline & Hipoacusia & $68-73^{*}$ \\
\hline & Atresia del conducto auditivo externo & \\
\hline \multirow[t]{5}{*}{ Ocular } & Quiste dermoide epibulbar & $19-28^{*}$ \\
\hline & Coloboma & $10-15$ \\
\hline & Microftalmia & 11 \\
\hline & Distrofia orbitaria & $15-43$ \\
\hline & Estenosis lagrimar & $11-14$ \\
\hline \multirow[t]{3}{*}{ Sistema óseo } & Malformaciones vertebrales (hemivértebra) & $44^{*}$ \\
\hline & Malformaciones costales (fusión costal) & * \\
\hline & Malformaciones en extremidades (hemimelia radial, pie equinovaro) & $7-16$ \\
\hline Cardiopatías congénitas & Defectos septales auriculares y ventriculares (lo más frecuente) & $15-25$ \\
\hline Sistema nervioso central & $\begin{array}{l}\text { Hipoplasia cerebral difusa, microcefalia, anomalías en nervios craneales VIII, VI y } \\
\text { VII, hidrocefalia, encefalocele, malformación de Arnold-Chiari, hipoplasia de cuerpo } \\
\text { calloso }\end{array}$ & $8-13$ \\
\hline Retraso en el neurodesarrollo & Lenguaje & $11^{*}$ \\
\hline Genitourinarias & $\begin{array}{l}\text { Riñón unilateral, agenesia, doble sistema colector, quiste renala , ectopia renal, } \\
\text { hidronefrosis, hidrouréter, entre otros }\end{array}$ & $10-13^{*}$ \\
\hline Gastrointestinales & Ano no permeable, atresia esofágica, entre otros & $2-12$ \\
\hline
\end{tabular}

aAnormalidades que presentó el paciente de este reporte.

posicionamiento: mantener la cabeza alineada con el tronco sostenida con los dedos pulgar e índice por la nuca y dar sostén con el resto de la mano en la espalda, en posición sentado sobre las piernas del cuidador, así como el uso de una tetina suave y alargada de flujo medio, posicionando el biberón a $90^{\circ}$ respecto a la cabeza. También se refirió a foniatría y rehabilitación para iniciar terapia de rehabilitación oral convencional (TRO), así como terapia con electroestimulación (NMES, neuromuscular electrical stimulation). El primer esquema de NMES consistió de 10 sesiones diarias (equipo VitalStim $®$ portátil con un generador de corriente que utiliza dos baterías $\mathrm{AA}$ ) con las siguientes especificaciones durante cada una: forma de onda, bifásica pulsada; frecuencia, $80 \mathrm{~Hz}$; duración de un ciclo, 700 milisegundos; duración de la terapia, 30 minutos; duración del ciclo durante la terapia, 60 segundos ( 57 segundos de actividad, 1 segundo rampa hacia abajo, 1 segundo apagado, 1 segundo rampa hacia arriba); intensidad, $10 \mathrm{~mA}$. Las almohadillas fueron colocadas a nivel del hueso hioides para estimular los músculos de la deglución con la intensidad ya mencionada. Durante el estímulo, el paciente practicó el tragado. En conjunto con la TRO se 


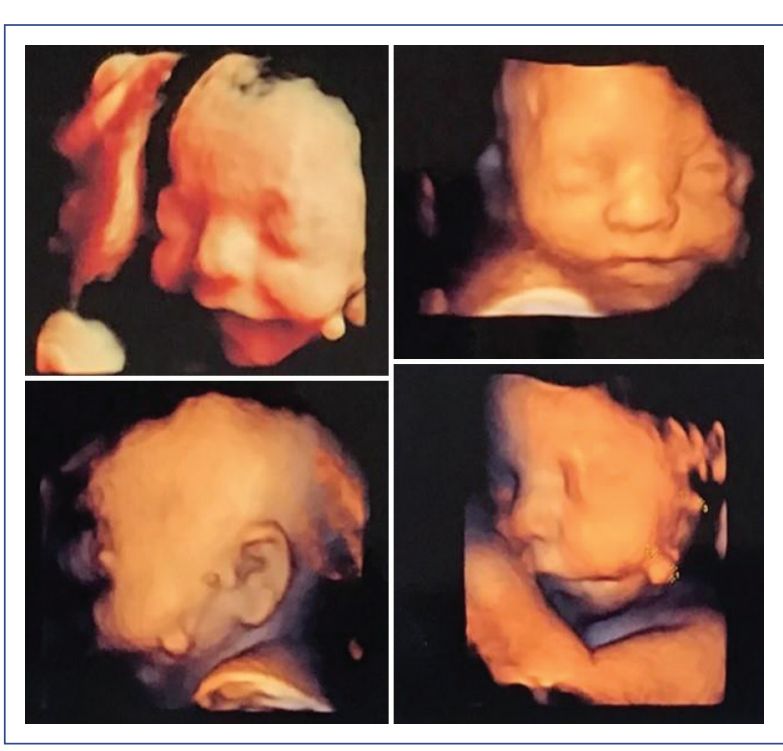

Figura 1. Ecografía prenatal a las 29 semanas de gestación, $1490 \mathrm{~g}$ de peso, polihidramnios, microrretrognatia, macrostomía y apéndices preauriculares bilaterales y maxilares del lado izquierdo.

realizaron técnicas sensitivas a base de masoterapia facial con los siguientes instrumentos: rodillo con textura rugosa, rodillo metálico para estimular sensación de temperatura, brocha de cerdas suaves para estimular el tacto fino y vibrador facial. En la cavidad oral se utilizaron cepillos dentales para bebé de marca comercial con diferentes texturas (cerdas suaves, de silicón cortas y largas), masaje digital con guante de látex durante la NMES masajeando los carrillos, las encías y el paladar según tolerancia. Al finalizar la terapia y durante los siguientes días los padres observaron una mejoría subjetiva: disminución de la frecuencia y la intensidad de la sialorrea y una mejor succión de la fórmula de inicio, aunque el sello labial no era evaluable por la presencia de defectos anatómicos (macrostomía). Antes de cada sesión se determinó la mejoría de la deglución con la escala ASHA (American SpeechLanguage Hearing Association) y la intensidad/frecuencia de la sialorrea con la escala diseñada por Thomas-Stonell y Greenberg5,6 (Fig. 3 y Tabla 2).

En el seguimiento por gastroenterología y nutrición pediátrica a los 4 meses de edad se registró una disminución de la velocidad del crecimiento, con peso de $5.25 \mathrm{~kg}$ (puntuación $Z<-2$ ), longitud de $59.5 \mathrm{~cm}$ (puntuación $Z<0$ ) y perímetro cefálico de $41 \mathrm{~cm}$ (puntuación $Z<+1$ ) (Fig. 2 A, B y D). Se observaron succión débil, sostén cefálico incompleto y atragantamiento intermitente, con datos clínicos evidentes de trastorno de la deglución. Se inició el espesamiento de la fórmula con cereal infantil y a los 6 meses se inició la introducción de alimentos en consistencia de papilla, con mínima aceptación. Presentó mejoría en la deglución y disminución de los episodios de atragantamiento, pero aún sin lograr cubrir los requerimientos energéticos para la edad. Por ello, a los 7 meses de edad se le colocó una sonda de gastrostomía mediante técnica abierta. Como hallazgo, se encontró un divertículo de Meckel.

A los 20 meses de edad se le realizó plastia de paladar, cierre de macrostomía y resección de los apéndices preauriculares. A los $23,24,25,27,30$ y 32 meses de edad recibió 10 terapias diarias de electroestimulación con una duración de 30 minutos cada una. Durante este periodo con sesiones de TRO y NMES mostró una mejoría paulatina en la succión, la deglución y la sialorrea (Fig. 3).

Desde los 7 meses hasta los 2 años y 6 meses de edad consumió la totalidad de la dieta por la sonda de gastrostomía. A partir de entonces comenzó el consumo por vía oral de leche y alimentos sólidos en consistencia de papilla de forma gradual hasta lograr la ingesta del $85 \%$ de los requerimientos energéticos por la boca, aún en consistencia de papilla y puré la mayor parte de los días a los 2 años y 8 meses de edad. Cuando no fue posible así, se complementó a través de la sonda de gastrostomía (Fig. 3). A la fecha es capaz de consumir todos los grupos de alimentos, a excepción de frutas por franco rechazo, y no ha presentado episodios de atragantamiento.

A partir de los 7 meses de edad (cuando logró una sedestación adecuada) y hasta la actualidad se recomienda a los padres una rutina de alimentación en los tres momentos de comida principales: sentado en una silla adecuada elegida únicamente para dicho proceso, conservando la cadera, las rodillas y los pies con apoyo a $90^{\circ}$; ofrecerle alimentos para estimulación oral (a pesar de la mínima aceptación); realizarle masoterapia con un cepillo de cerdas suaves y un utensilio metálico para estimular la temperatura en las mejillas, perioral (músculo orbicular de la boca), los carrillos orales, el paladar y la lengua, y masoterapia con cepillo dental para bebé. Todo lo anterior con una frecuencia de tres veces al día, 10 repeticiones y según la tolerancia del paciente.

\section{Discusión}

En este caso se presentan los resultados exitosos del manejo de la disfagia orofaríngea con TRO 


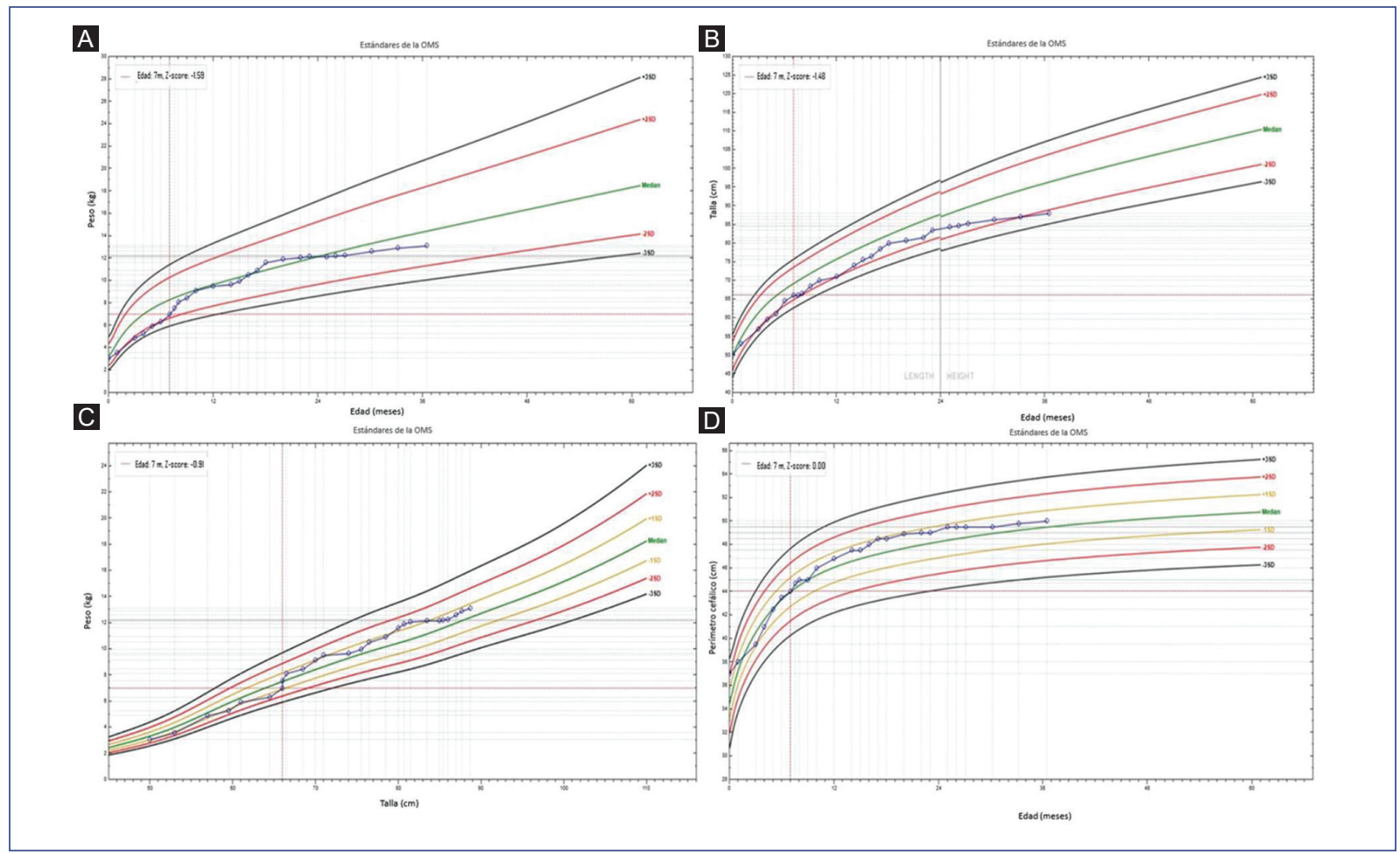

Figura 2. Evolución antropométrica según los estándares del crecimiento de la Organización Mundial de la Salud. A los 3 meses (tercera medición) se inició la terapia de rehabilitación oral. A los 7 meses se colocó sonda de gastrostomía. A: peso para la edad. B: longitud para la edad. C: peso para la longitud. D: perímetro cefálico para la edad.

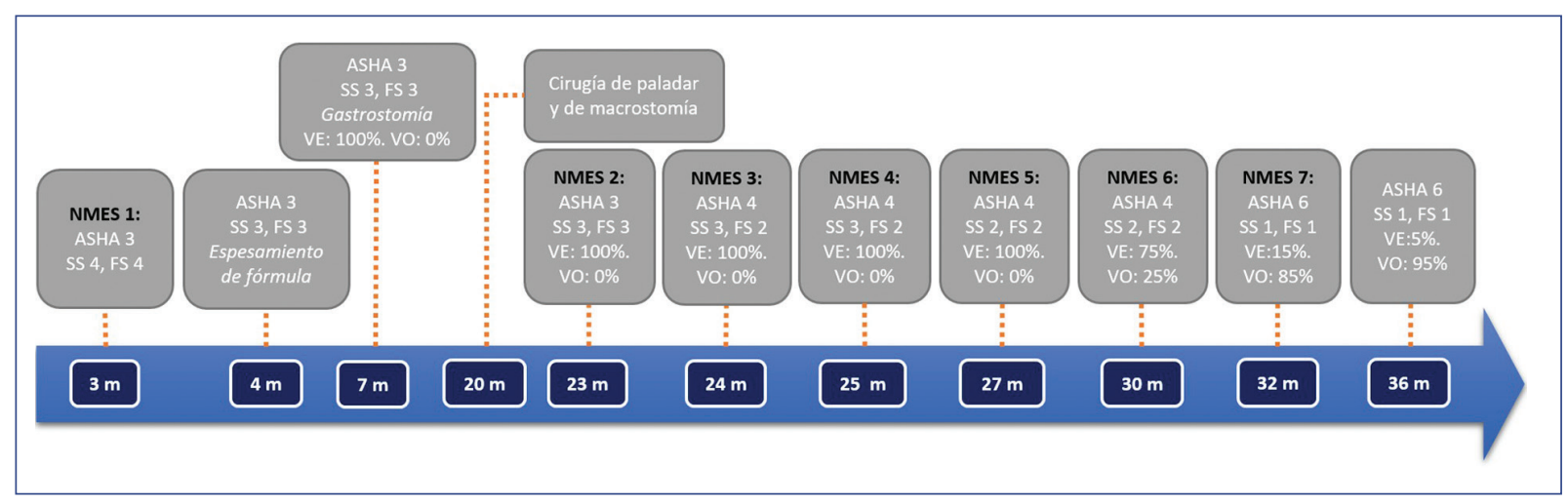

Figura 3. Evolución clínica de la gravedad del trastorno de la deglución y de la sialorrea durante las intervenciones terapéuticas: espesamiento de fórmula, gastrostomía, cirugía y terapia de electroestimulación. ASHA: American Speech-Language Hearing Association; FS: frecuencia de la sialorrea (1, nunca; 2, ocasional; 3, frecuente; 4, constante); m: meses; NMES: electroestimulación neuromuscular (10 sesiones diarias de 30 minutos cada una de $10 \mathrm{~mA}$ de intensidad); SS: severidad de la sialorrea (1, seco; 2, leve; 3, moderado; 4, severo; 5, profuso); VE: porcentaje de requerimientos calóricos por vía enteral (por sonda de gastrostomía); V0: porcentaje de requerimientos calóricos por vía oral.

convencional, NMES, plastia palatina y alimentación a través de sonda de gastrostomía en un paciente con síndrome de Goldenhar que presentó falla para crecer.
La falta de crecimiento ponderal se deriva de un trastorno de la deglución asociado al síndrome de Goldenhar, el cual tiene dos ejes: el primero, el defecto anatómico del paladar y del maxilar (paladar hendido, 
Tabla 2. Escala ASHA (American Speech-Language Hearing Association) para la evaluación de la deglución

\section{Nivel Características}

1 El individuo no puede tragar nada de forma segura por vía oral.

Toda la nutrición e hidratación se recibe por medios no orales.

2 El individuo no puede deglutir de manera segura para nutrición e hidratación, pero puede tomar/ingerir ciertas consistencias con señales máximas regulares solo durante la terapia. Se requiere un método alternativo de alimentación.

3 Se requiere un método alternativo de alimentación, ya que el individuo toma menos del $50 \%$ de la nutrición e hidratación por vía oral o la deglución es segura con el empleo regular de señales moderadas para usar estrategias compensatorias o requiere restricciones de dieta máximas.

$4 \quad$ La deglución es segura, pero generalmente requiere señales moderadas para usar estrategias compensatorias o el individuo tiene restricciones moderadas en la dieta o aún requiere alimentación por sonda y suplementos orales.

$5 \quad$ La deglución es segura con restricciones de dieta mínimas u ocasionalmente requiere indicaciones mínimas para usar estrategias compensatorias. De vez en cuando puede señalar para sí mismo. Todas las necesidades de nutrición e hidratación se satisfacen por vía oral al comer.

$6 \quad$ La deglución es segura y el individuo come y bebe de forma independiente, y rara vez requiere una señal mínima. Por lo general, señala para sí mismo cuando se presentan dificultades. Es posible que deba evitar alimentos específicos o que requiera tiempo adicional (debido a la disfagia).

7 La capacidad del individuo para comer independientemente no está limitada por la función de deglución. La deglución es segura y eficiente para todas las consistencias. Las estrategias compensatorias se usan efectivamente cuando es necesario.

Traducida de Kim, et al. ${ }^{6}$

reportado en el $16-20 \%$ de los casos); el segundo, un funcionamiento anómalo de los músculos de la masticación y la deglución por un desarrollo anormal de los nervios craneales $\mathrm{V}$ y VII, en su mayoría por defectos en el foramen oval ${ }^{1,2,7}$. Lo anterior, a consecuencia de un desarrollo anormal del primero y segundo arcos faríngeos ${ }^{2}$.

Por otro lado, se ha descrito que del $40-80 \%$ de los pacientes con microsomía hemifacial presentarán dificultades para la succión, la masticación y la deglución ${ }^{8}$. En 2017, van de Lande, et al. ${ }^{8}$ reportaron más dificultades para la formación del bolo alimenticio, y por lo tanto para la deglución, en los pacientes con hipoplasia mandibular grave. En el presente reporte se describe un paciente con varios factores asociados con la alteración de la mecánica de la deglución: paladar hendido, macrostomía, hipoplasia mandibular y alteraciones en la función de los músculos de la masticación y la deglución. Es bien conocido que una alteración en la mecánica de la deglución no tratada de manera oportuna invariablemente repercutirá en la velocidad de crecimiento. En el caso descrito se observó que la velocidad de crecimiento disminuyó significativamente desde el primer mes de edad, y no fue sino hasta los 3 meses de vida extrauterina que se inició el manejo de rehabilitación.

No es de nuestro conocimiento la existencia de reportes en los que se haya utilizado TRO y NMES para mejorar la deglución en pacientes con síndrome de Goldenhar. Únicamente se han descrito la corrección quirúrgica de los defectos anatómicos y la alimentación por sonda nasogástrica o por gastrostomía como abordaje para la recuperación del estado nutricional en los pacientes con síndrome de Goldenhar ${ }^{2}$. El presente caso se trató conforme a lo establecido en la literatura: alimentación por sonda (7 meses de edad) y corrección de los defectos anatómicos (20 meses de edad). Adicionalmente, como primera intervención médico-nutricia (4 meses de edad) se optó por el espesamiento de la fórmula para proveer una mayor densidad energética, así como para disminuir los episodios de atragantamiento.

Con la TRO y la NMES se observó mejoría en la deglución y en la intensidad de la sialorrea a los 3 meses de edad, lo cual pudo contribuir a que el espesamiento de la fórmula diera mejores resultados a los 4 meses de edad. En la curva de peso para la edad (Fig. 2 A) se logró alcanzar una puntuación Z de -2 (3-6 meses de edad) antes de la colocación de la sonda de gastrostomía (7 meses). De los 7 a los 20 meses de edad (plastia palatina y cierre de macrostomía), periodo durante el cual fue alimentado por sonda como única intervención, se observaron variaciones en la curva de crecimiento por ajuste en kilocalorías de la dieta. Sin embargo, no hubo cambios en la deglución ni en la sialorrea (ASHA 3 y sialorrea frecuente de intensidad moderada) durante este periodo (Fig. 3). Finalmente, la deglución (ASHA 4 y sialorrea ocasional) mejoró sustancialmente en el paciente hasta los 24 meses de edad y con el tercer grupo de sesiones de NMES (30 en total). Cabe señalar que no se observaron cambios en la antropometría influidos por esta terapia, ya que el aporte energético fue administrado en su totalidad por 
sonda. Hasta la séptima sesión de NMES (70 en total) mejoró sustancialmente la deglución (ASHA 6 y sin sialorrea) (Fig. 3). En ese momento, el paciente comenzó a incrementar la ingesta por vía oral, con apoyo de la sonda para aquellos alimentos que rechazaba (frutas). La antropometría se mantuvo constante entre los 32 y los 36 meses de edad (Fig. 2 A).

Los terapeutas expertos en foniatría y lenguaje realizan la terapia de rehabilitación motora oral, centrándose en el apoyo a la nutrición y el desarrollo de habilidades de alimentación, así como en garantizar la deglución segura para la prevención de la neumonía por aspiración y la asfixia ${ }^{7-9}$. Dicha terapia se clasifica en movimientos activos, pasivos (TRO) y sensitivos (con o sin NMES). Los primeros incluyen una serie de movimientos realizados para que el paciente adquiera fuerza (como ejercicios para fortalecer la lengua) y aumente la resistencia y la potencia muscular. La evidencia sobre su eficacia es débil e insuficiente para determinar con certeza los efectos sobre estas habilidades en pacientes pediátricos con trastornos de la deglución. Los ejercicios pasivos pueden incluir masajes, caricias, estiramientos y presiones, aunque tampoco existe evidencia que respalde su eficacia. La terapia sensitiva consiste en la aplicación de calor, frío, vibración de alta frecuencia o NMES $^{8-10}$. Se han observado mejores resultados con la combinación de TRO y NMES por la estimulación de los dos tipos de fibras musculares existentes, tipo I y tipo II, respectivamente ${ }^{11}$.

La terapia con NMES, que se realizó por primera vez en 1996, es un tratamiento propuesto para la disfagia que implica la estimulación eléctrica de los músculos de la deglución para mejorar la fuerza muscular y la coordinación con el objetivo de estimular la musculatura de la laringe y que esta ascienda en la segunda fase de la deglución. Fue aprobada por la Food and Drug Administration y comercializada por Chattanooga Group bajo el nombre de VitalStim ${ }^{\circledR 1}$. La hipótesis consiste en que los impulsos eléctricos pueden mejorar la fuerza muscular faríngea en combinación con la reorganización cortical. Sin embargo, existe poca evidencia que la respalde, y aún es más escasa en población pediátrica. Por otro lado, resulta más contundente en pacientes con disfagia adquirida que en aquellos con disfagia de origen primario 8 ,12.

Existen estudios en adultos que demuestran la eficacia en pacientes que recibieron la terapia después de un accidente cerebrovascular ${ }^{10}$. En lo que respecta a la población pediátrica, se ha utilizado en pacientes con daño neurológico con resultados positivos ${ }^{5}$. La técnica consiste en colocar electrodos en la piel (parte anterior del cuello), los cuales liberarán pequeñas cantidades de energía para estimular los músculos encargados de la deglución ${ }^{12}$. Lamentablemente, existen pocos artículos de investigación que reporten la eficacia de la NMES en pediatría y la evidencia es insuficiente.

Por un lado, Frost, et al. ${ }^{7}$ reportaron más beneficios con la terapia de electroestimulación en los pacientes con disfagia adquirida en comparación con aquellos con disfagia primaria. Por otro lado, Marcus, et al. ${ }^{12}$ reportaron que dicha terapia sí resultó benéfica en el tratamiento de la disfagia en pacientes pediátricos. Otro estudio de casos y controles en población pediátrica reportó una mejoría con el uso de ambas terapias, aunque únicamente cuando se utilizó NMES se observó una diferencia significativa ${ }^{5}$.

En una serie de casos, Shaw, et al. ${ }^{11}$ describieron que se requieren entre 5 y 28 sesiones para mostrar mejoría en la deglución. Nuestro paciente mostró mejoría en la sialorrea después de la primera terapia de 10 sesiones. Sin embargo, no fue sino hasta el tercer ciclo de 10 sesiones (30 sesiones) que la deglución mejoró (Fig. 3).

Con respecto al estado nutricional, se puede asumir que la recuperación nutricional del paciente se debió principalmente a la alimentación enteral a través de la sonda de gastrostomía, puesto que incrementó la velocidad de crecimiento a partir de los 7.5 meses de edad, es decir, posterior a la colocación de la sonda. Aunque el paciente no ha logrado un sello labial adecuado, incluso después de la cirugía, comenzó a aceptar alimentos con consistencia de papilla por vía oral tras las primeras sesiones que fueron deglutidos de manera exitosa (sin asfixia ni atragantamientos) tras varias terapias de rehabilitación. Además, mostró un avance constante en la escala ASHA hasta lograr la ingesta por vía oral en su totalidad y sin complicaciones (Fig. 3). Según el avance mantenido en la alimentación total por vía oral, se planteará retirar la sonda de gastrostomía de forma definitiva.

Hasta donde se conoce, este es el primer caso reportado en la literatura de un paciente con síndrome de Goldenhar y falla para crecer a consecuencia de una mala deglución que se manejó con alimentación enteral mediante sonda de gastrostomía, TRO y NMES. Existen varios estudios que evidencian la eficacia de dichas terapias de rehabilitación en pacientes adultos con disfagia. En cambio, para la población pediátrica aún existe muy poca evidencia.

El paciente de este reporte actualmente ingiere la totalidad de sólidos y líquidos por vía oral. Con el nivel bajo de evidencia científica que este caso aislado aporta, se concluye que la terapia de rehabilitación en conjunto con la terapia convencional, aunado a la 
corrección anatómica, dio resultados positivos para el trastorno de la deglución.

\section{Responsabilidades éticas}

Protección de personas y animales. Los autores declaran que para esta investigación no se han realizado experimentos en seres humanos ni en animales.

Confidencialidad de los datos. Los autores declaran que han seguido los protocolos de su centro de trabajo sobre la publicación de datos de pacientes.

Derecho a la privacidad y consentimiento informado. Los autores han obtenido el consentimiento informado de los pacientes o individuos referidos en el artículo. Este documento obra en poder del autor de correspondencia.

\section{Conflicto de intereses}

Los autores declaran no tener ningún conflicto de intereses.

\section{Financiamiento}

Ninguno.

\section{Bibliografía}

1. Beleza-Meireles $A$, Hart R, Clayton-Smith J, Oliveira R, Reis CF, Venâncio $\mathrm{M}$, et al. Oculo-auriculo-vertebral spectrum: clinical and molecular analysis of 51 patients. Eur J Med Genet. 2015;58:455-65.

2. Bogusiak K, Puch A, Arkuszewski P. Goldenhar syndrome: current perspectives. World J Pediatr. 2017;13:405-15.

3. Beleza-Meireles A, Clayton-Smith J, Saraiva JM, Tassabehii M. Oculo-auriculo-vertebral spectrum: a review of the literature and genetic update. J Med Genet. 2014;51:635-45.

4. Manara R, Brotto D, Ghiselli S, Mardari R, Toldo I, Schifano G, et al. Cranial nerve abnormalities in oculo-auriculo-vertebral spectrum. Am J Neuroradiol. 2015;36:1375-80.

5. Thomas-Stonell N, Greenberg J. Three treatment approaches and clinical factors in the reduction of drooling. Dysphagia. 1988;3:73-8

6. Kim J, Oh BM, Kim JY, Lee GJ, Lee SA, Han TR. Validation of the videofluoroscopic dysphagia scale in various etiologies. Dysphagia. 2014;29:438-43.

7. Frost J, Robinson HF, Hibberd J. A comparison of neuromuscular electrical stimulation and traditional therapy, versus traditional therapy in patients with longstanding dysphagia. Curr Opin Otolaryngol Head Neck Surg. 2018;26:167-73.

8. van de Lande LS, Caron CJJM, Pluijmers BI, Joosten KFM, Streppel M, Dunaway DJ, et al. Evaluation of swallow function in patients with craniofacial microsomia: a retrospective study. Dysphagia. 2018;33:234-42.

9. Kiger M, Brown CS, Watkins L. Dysphagia management: an analysis of patient outcomes using VitalStim therapy compared to traditional swallow therapy. Dysphagia. 2006;21:243-53.

10. Mejía J, Baez P. Satisfacción en los cuidadores primarios de los pacientes con parálisis cerebral posterior al tratamiento con VitalStim®. Medicina e Investigación. 2015;3:35-42.

11. Shaw GY, Sechtem PR, Searl J, Keller K, Rawi TA, Dowdy E. Transcutaneous neuromuscular electrical stimulation (VitalStim) curative therapy for severe dysphagia: myth or reality? Ann Otol Rhinol Laryngol. 2007;116:36-44.

12. Marcus S, Friedman JN, Lacombe-Duncan A, Mahant S. Neuromuscular electrical stimulation for treatment of dysphagia in infants and young children with neurological impairment: a prospective pilot study. BMJ. 2019;3:e000382. 